\title{
Preoperative Lymph Node Staging by FDG PET/CT With Contrast Enhancement for Thyroid Cancer: A Multicenter Study and Comparison With Neck CT
}

\author{
Ari Chong ${ }^{1, \star} \cdot$ Jung-Min Ha ${ }^{1, \star} \cdot$ Yeon-Hee Han ${ }^{2}$ Eunjung Kong ${ }^{3} \cdot$ Yunjung Choi ${ }^{4} \cdot \mathrm{Ki} \mathrm{Hwan} \mathrm{Hong}^{5} \cdot$ Jun-Hee Park ${ }^{6}$ \\ Sung Hoon $\mathrm{Kim}^{7} \cdot$ Jung Mi Park ${ }^{8}$
}

\begin{abstract}
${ }^{1}$ Department of Nuclear Medicine, Chosun University Hospital, Gwangju; ${ }^{2}$ Department of Nuclear Medicine, Chonbuk National University Hospital, Jeonju; ${ }^{3}$ Department of Nuclear Medicine, Yeungnam University Medical Center, Daegu; ${ }^{4}$ Department of Nuclear Medicine, Hallym University Sacred Heart Hospital, Anyang; ${ }^{5}$ Department of Otolaryngology-Head and Neck Surgery, Research Institute of Clinical Medicine, Chonbuk National University Hospital, Chonbuk National University Medical School, Jeonju; ${ }^{6}$ Department of Otolaryngology-Head and Neck Surgery, Chosun University College of Medicine, Gwangju, 'Department of Radiology, Seoul St. Mary's Hospital, College of Medicine, The Catholic University of Korea, Seoul; ${ }^{8}$ Department of Nuclear Medicine, Soonchunhyang University Bucheon Hospital, Bucheon, Korea
\end{abstract}

Objectives. The purpose of this study was to compare lymph node (LN) staging using ${ }^{18} \mathrm{~F}$-fluorodeoxyglucose (FDG) positron emission tomography/computed tomography (PET/CT) with contrast-enhancement (CE) PET/CT and contrastenhanced neck CT (neck CT) in patients with thyroid cancer with level-by-level comparison with various factors.

Methods. This was a retrospective multicenter study. A total of 85 patients were enrolled. Patients who underwent a preoperative evaluation by CE PET/CT and neck CT for thyroid cancer were enrolled. The gold standard for LN was the combination of surgical pathology and clinical follow-up. We compared CE PET/CT with neck CT using a level-bylevel method. Factors, including age, sex, camera, arm position, tumor size, extra-thyroidal extension, tumor location, number of primary tumors, primary tumor maximum standardized uptake value, and the interval from scan to operation were also analyzed.

Results. Overall accuracy was $81.2 \%$ for CE PET/CT and $68.2 \%$ for neck CT. CE PET/CT was more sensitive than neck CT $(65.8 \%$ vs. $44.7 \%)$. Also, CE PET/CT showed higher negative predictive value $(77.2 \%$ vs. $66.1 \%)$. CE PET/CT showed good agreement with the gold standard (weighted kappa [ $\mathrm{\kappa}], 0.7)$ for differentiating N0, N1a, and N1b, whereas neck CT showed moderate agreement (weighted $\kappa, 0.5$ ). CE PET/CT showed better agreement for the number of levels involved with the gold standard (weighted $\kappa, 0.7$ ) than that of neck CT with the gold standard (weighted $\kappa, 0.5$ ). The accuracies for differentiating N0, N1a, and N1b were $81.2 \%$ for CE PET/CT and $68.2 \%$ for neck CT. Level-by-level analysis showed that CE PET/CT was more sensitive and has higher negative predictive value for detecting ipsilateral level IV and level VI LNs than neck CT. Other analyzed factors were not related to accuracies of both modalities.

Conclusion. CE PET/CT was more sensitive and reliable than neck CT for preoperative LN staging in patients with thyroid cancer.

Keywords. Positron-Emission Tomography; Fluorodeoxyglucose F18; Computed Tomography; Thyroid Neoplasms

- Received September 15, 2015

Revised December 31, 2015

Accepted March 11, 2016

- Corresponding author: Jung Mi Park

Department of Nuclear Medicine, Soonchunhyang University Bucheon

Hospital, 170 Jomaru-ro, Wonmi-gu, Bucheon 14584, Korea

Tel: +82-32-621-5884, Fax: +82-32-621-5749

E-mail: jmipark@schmc.ac.kr

*The first two authors contributed equally to this study.

\section{INTRODUCTION}

Patients with thyroid cancer generally have a good prognosis, and 5-year overall survival is $>90 \%[1,2]$. However, cervical lymph node (LN) metastasis occurs in $30 \%$ to $80 \%$ of patients with thyroid cancer [3]. LN metastasis is associated with an in-

Copyright @ 2017 by Korean Society of Otorhinolaryngology-Head and Neck Surgery

This is an open-access article distributed under the terms of the Creative Commons Attribution Non-Commercial License (http://creativecommons.org/licenses/by-nc/4.0)

which permits unrestricted non-commercial use, distribution, and reproduction in any medium, provided the original work is properly cited. 
creased risk of locoregional recurrence, even in low-risk patients [3]. Therefore, appropriate $\mathrm{LN}$ dissection is very important. Adam et al. [4] reported that cervical LN metastasis is associated with compromised survival in young patients with papillary cancer. LN metastases are most common at cervical levels VI and VII [5]. Lateral neck metastases (levels I to V) are less common but may be associated with a worse prognosis [5,6]. LN dissection is indicated for cases of LN metastasis [7]. Prophylactic LN dissection can also be performed, although it is controversial $[3,8]$. However, the extent of $\mathrm{LN}$ dissection can directly affect the quality of life due to cosmetic issues, postoperative complications, and postoperative morbidities [3,5]. Therefore, a preoperative neck evaluation for thyroid cancer is necessary to determine the extent of the surgical resection $[9,10]$. Imaging modalities, such as neck ultrasonography (US), computed tomography (CT), positron emission tomography (PET)/CT, and magnetic resonance imaging are important modalities for assessing the lateral neck [5]. The type of neck dissection to be performed depends on the cervical level. Determining the cervical level(s) involved is important to decide the extent of dissection.

Byun et al. [11] reported that addition of ${ }^{18} \mathrm{~F}$-fluorodeoxyglucose (FDG) uptake information to ultrasonography results can improve the sensitivity of metastatic LN detection. Choi et al. [12] reported that different results according to cervical levels in evaluation of LN between CT and US. In their study, compared to US, CT showed better sensitivity in detecting central node (level VI) but lower sensitivity in detecting lateral nodes (level II to $\mathrm{V}$ ) [12]. We thought the performances in preoperative $\mathrm{LN}$ staging might be different between PET/CT and contrast-enhanced neck CT (neck CT) according to level-by-level comparison as well as other factors like FDG uptake.

In this study, we compared the preoperative LN staging of contrast-enhanced (CE) FDG PET/CT and neck CT in patients with thyroid cancer in a level-by-level analysis with other factors.

\section{MATERIALS AND METHODS}

\section{Patient selection}

This was a retrospective study. We enrolled patients with thyroid papillary carcinoma who underwent both CE PET/CT and neck CT before surgery for thyroid cancer from January 2010 to May

\section{H I G H L I G H T S}

- ${ }^{18} \mathrm{~F}$-fluorodeoxyglucose positron emission tomography/computed tomography with contrast-enhancement (CE PET/CT) was more accurate than CT for differentiating N0, N1a, and N1b $(81.2 \%$ vs. $68.2 \%)$.

- For ipsilateral level IV and IV, CE PET/CT was more sensitive and has higher negative predictive value than $\mathrm{CT}$.
2014 at four institutions.

All enrolled patients underwent thyroid surgery with or without neck dissection. The interval between surgery and CE PET/ CT was $<3$ months. Patients were excluded if the interval between CE PET/CT and neck CT was $>1$ month. Patients were excluded if the interval between the operation and imaging was $>1$ month. This protocol was approved by the Institutional Review Boards at each hospital.

\section{${ }^{18} \mathrm{~F}$-FDG PET/CT and CE CT}

$\mathrm{PET} / \mathrm{CT}$ scans were acquired after a single FDG injection. Patients fasted for 6 hours before the ${ }^{18} \mathrm{~F}-\mathrm{FDG}$ injection (serum glucose level $<180 \mathrm{mg} / \mathrm{dL}$ ). FDG dose was corrected for body mass index at all centers. The scan was obtained from the subcranial region to the upper thigh (torso) 60 to 90 minutes after the injection. A low-dose $\mathrm{CT}$ acquisition without contrast enhancement (NE CT) was initiated first, followed by PET acquisition. Then, the CE CT scans were collected. Iterative reconstruction was done using ordered-subset expectation maximization software. The attenuation was corrected by NE CT. NE PET/CT was not performed at one institution, and CE PET/CT was used for attenuation correction in these patients $(n=14)$.

The PET/CT machines used in this study were as follows: the Biograph TruePoint 40 PET/CT scanner (Siemens Medical Solutions, Knoxville, TN, USA) or the Biograph 16 PET/CT scanner (Siemens Medical Solutions), the Discovery ST PET/CT instrument (GE Medical Systems, Milwaukee, WI, USA), the Discovery ST PET/CT instrument (GE Medical Systems), the Discovery VCT PET/CT instrument (GE Medical Systems), and the Gemini TF (Philips-ADAC Medical Systems, Cleveland, OH, USA). The workstations used for reconstruction were the Syngo multimodality workplace, Exeleris Advanced Workstation 4.4 (GE Medical Systems).

\section{CE neck CT}

Neck CT was performed as preoperative staging. The scanners used at the various institutions were: the Somatom Sensation 16 (Siemens Medical Solutions, Erlangen, Germany), the MX 8000 IDT 16 (Philips Medical Systems, Eindhoven, the Netherlands), and MX 8000 Infinite Detector Technology (Philips Medical Systems).

\section{Image analysis and information obtained}

Each scan was read by qualified nuclear physicians and radiologists at each institution. The report of each institution was analyzed retrospectively without additional reading. The following primary tumor information was obtained from the neck CT report: size (cm), extrathyroid extension (positive/negative), and number of primary tumors. The same information was obtained from the CE PET/CT report but the primary tumor maximum standardized uptake value (SUVmax) was measured. When tumors were bilateral, we used the highest SUVmax value avail- 
able. The LN analysis was undertaken on a level-by-level basis, using the cervical LN levels described in the American Joint Committee on Cancer staging manual [13].

Information about the metastatic LN levels and LN stages (N0, N1a, or N1b) was obtained from neck CT. LNs with a long axis diameter $(>1 \mathrm{~cm}$ or $>1.5 \mathrm{~cm}$ for submandibular or jugulodiagastric LNs) were considered metastatic nodes $[14,15]$. In addition, central lucency suggesting necrosis was considered a malignant feature, regardless of size [14,15]. Borderline sized LNs with pathologic contrast enhancement were also considered metastatic [14,15].

The metastatic LN levels and LN stages (N0, N1a, or N1b) were determined from PET/CT. LNs with SUVmax $>2.0$ were considered metastatic nodes $[15,16]$. In addition, central lucency suggesting necrosis was considered a malignant feature, regardless of SUVmax $[14,15]$. However, uptake by tissues other than nodes, such as by a vessel, was considered benign after review of the CT portion of the CE PET/CT scan, regardless of SUVmax [15]. In addition, LNs with pathologic contrast enhancement were considered metastatic, regardless of SUVmax.

For determination of ipsilateral or contralateral side of the neck, the location of primary tumor was used. When tumors are at both sides of the thyroid lobes, metastatic LNs on either sides of the neck were considered as "ipsilateral." Clinical information such as age, sex, interval from PET to the operation, or interval from CT to PET, camera type, and PET/CT arm position was also analyzed.

\section{Gold standard for the final diagnosis}

The LN analysis was performed on a level-by-level basis. The final diagnosis for LNs that were not dissected out during the operation was determined by follow-up of serum thyroglobulin or US or postoperative radioactive iodine scan (Table 1). Because the purpose of the clinical follow-up is to confirm the status of LNs at the timing of preoperative evaluation, the follow-up period was limited to 6 months after surgery.

\section{Statistical analysis}

Accuracy, sensitivity, specificity, positive predictive value, and negative predictive value (NPV) were analyzed for all cervical LN levels and for the cervical levels detected on CE PET/CT and neck CT. Analyses were done to differentiate between N0 and N1 and to differentiate between N0, N1a, and N1b. The comparison of the number of involved cervical levels between CE PET/CT and neck CT was done using the paired $t$-test and degree of agreement. We analyzed the effects of clinical factors, such as sex, scanner type, arm position, $\mathrm{T}$ staging, extrathyroidal extension, and pathological tumor type, on the accuracies of $\mathrm{CE}$ PET/CT and neck CT using Pearson chi-square test. Quantitative data, such as age, tumor size, number of primary tumors, and primary tumor SUVmax were analyzed by logistic regression, the $t$-test, or the Mann-Whitney test. For analysis of clinical
Table 1. Patient characteristics $(n=85)$

\begin{tabular}{lc}
\hline Variable & No. (\%) \\
\hline No. of patients (center A:B:C:D) & $53(62): 14(17): 13(15): 5(6)$ \\
Sex (male:female) & $14(17): 71(84)$ \\
Age (yr), median (range) & $54(19-80)$ \\
CE PET/CT arm position (up:down) & $54(64): 31(37)$ \\
Clinical follow-up methods to confirm & \\
Tg+US & $23(27)$ \\
Tg+US+RI & $14(17)$ \\
Tg+US+RI+biopsy & $2(2)$ \\
Tg+RI & $17(20)$ \\
Tg & $29(34)$ \\
Preoperative staging (NO:N1a:N1b) & $62(73): 5(6): 18(21)$ \\
By neck CT & $57(67): 9(11): 19(22)$ \\
By CE PET/CT & $30(35): 2(2): 48(57): 5(6)$ \\
Pathologic results at operation & $25(29): 33(39): 27(32)$ \\
pT1:pT2:pT3:pT4 & \\
Tumor side (left:right:both) & \\
Final results of follow-up of lymph node & \\
N0:N1a:N1b & $47(55): 18(21): 20(24)$ \\
Central lymph node metastasis & $34(38)$ \\
only, no. of patients (level) & $19: 2: 0$ \\
Lateral lymph node metastasis & \\
(ipsilateralb):bilateral: & \\
contralateral only) & \\
Overall stage (l:Il:Ill:IVA) & $(29): 0(0): 38(45): 22(26)$ \\
\hline
\end{tabular}

CE PET/CT, ${ }^{18}$ F-fluorodeoxyglucose positron emission tomography/computed tomography with contrast-enhancement; Tg, serum level of thyroglobulin; US, neck ultrasonography; RI, radioactive iodine whole body scan.

${ }^{\text {a) }}$ Clinical follow-up was done within 6 months postoperatively. ${ }^{\text {b) For tumors }}$ in both sides of thyroid lobes, metastatic lymph nodes in either sides of the neck were considered as ipsilateral metastasis. In analysis of ipsilateral metastasis, central lymph nodes (level VI and VII) were excepted. There was no case with lymph node metastasis to contralateral neck without ipsilateral side metastasis. ${ }^{c}$ TNM staging based on the seventh edition of the American Joint Committee on Cancer. Final staging based on combined pathological and clinical follow-up results, which were obtained within 6 months of the operation.

factors, we used groups of "correct/incorrect" cases of each modalities based on the final results. For example, when CE PET/ CT successfully staged the $\mathrm{LN}(\mathrm{N} 0, \mathrm{~N} 1 \mathrm{a}$, and $\mathrm{N} 1 \mathrm{~b})$ in some patients, they were categorized as CE PET/CT correct cases.

The sensitivity and specificity comparisons were performed using the McNemar test. To compare predictive values, we used R Core Team (R Foundation for Statistical Computing, Vienna, Austria; https://www.R-project.org/). We also used package DTComPair for R (R package ver. 1.0.3; http://CRAN.R-project. org/package=DTComPair). Also, Medcalc ver. 13.3.3.0 (Medcalc Inc., Ostend, Belgium), and IBM SPSS ver. 19.0 (IBM Co., Armonk, NY, USA) were used.

\section{RESULTS}

Eighty-five patients were enrolled from four institutions (Fig. 1). 

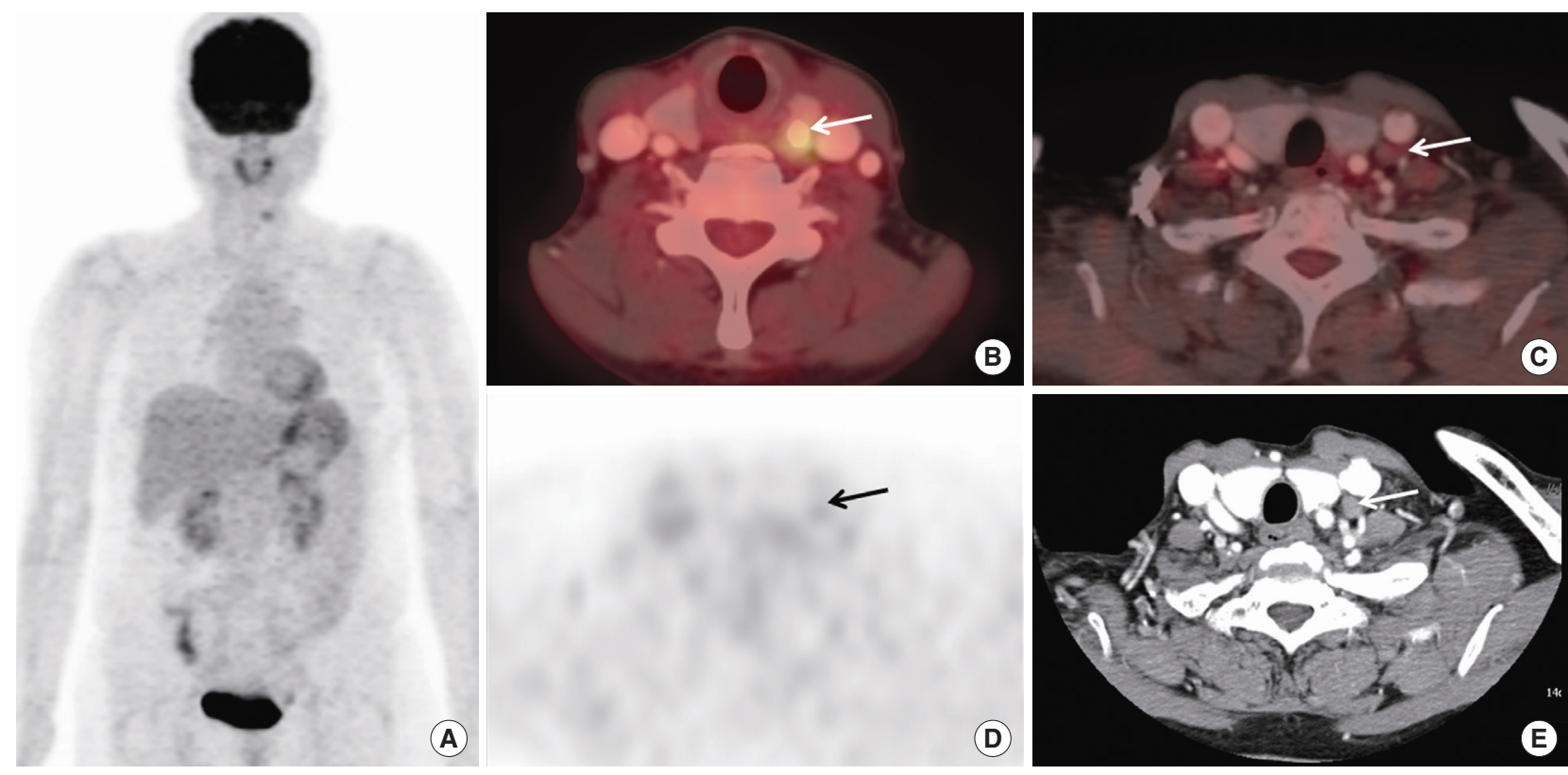

(A)
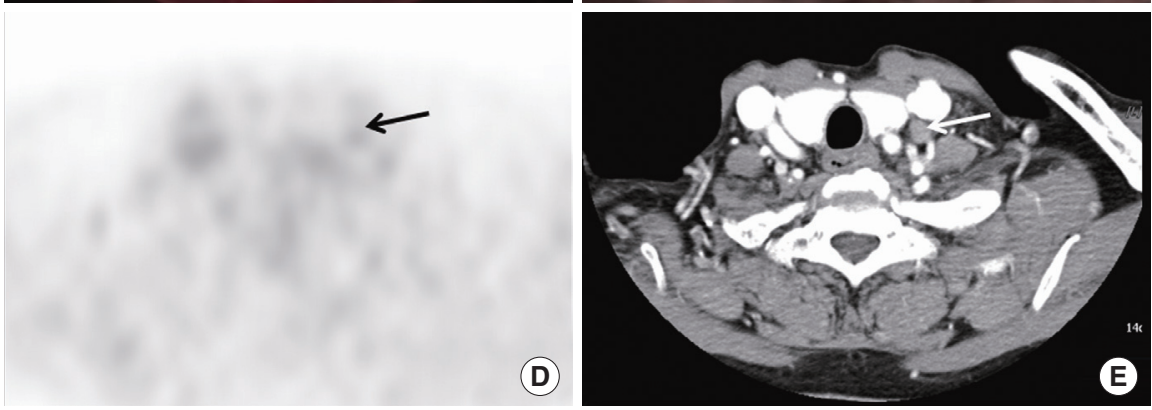

Fig. 1. A case of different finding of ${ }^{18} \mathrm{~F}$-fluorodeoxyglucose positron emission tomography/computed tomography with contrast-enhancement (CE PET/CT) and contrast-enhanced neck CT (neck CT) in a preoperative lymph node staging in a patient with thyroid cancer. A 56-year-old female patient underwent CE PET/CT and neck CT with contrast-enhancement before thyroid cancer operation: (A) maximum intensity image of CE PET/CT, (B, C) axial fusion images, (D) axial PET only image at the same level of $C$, and (E) axial image of neck CT. A calcified nodule with focal hypermetabolism with maximum standardized uptake value (SUVmax) 4.2 was found in left thyroid lobe which was proven to be papillary cancer by fine needle aspiration (arrow in B). Both of CE PET/CT and neck CT showed a lymph node adjacent to left carotid vessel (arrows in C-E). The long diameter by neck CT was about $1 \mathrm{~cm}(\mathrm{E})$, suggesting metastatic lymph node. However, FDG uptake was not high (arrows in D, SUVmax 1.4), suggesting benign feature. After operation, biopsy revealed benign lymph node. Also, clinical follow-up with radioactive iodine scan and neck ultrasonography (images not shown), there was no metastatic lymph node.

The patients' characteristics are described in Table 1. Average SUVmax of primary tumor was $9.5 \pm 11.3 \mathrm{~cm}$ (range, 1.5 to $50.7 \mathrm{~cm})$ and average size of primary tumor was $1.13 \pm 0.9 \mathrm{~cm}$ (range, 0.2 to $5.0 \mathrm{~cm}$ ).

\section{Analysis by patients}

The overall accuracy for detecting a cervical LN metastasis (differentiate between N0 and N1) was $81.2 \%$ for CE PET/CT and $68.2 \%$ for neck CT. CE PET/CT was more sensitive for detecting overall cervical LN metastases than that of neck CT $(65.8 \%$ vs. $44.7 \%, P=0.008$ ) (Table 2). Also, CE PET/CT showed significantly higher NPV than CT (77.2\% vs. $66.1 \%, P=0.001)$.

The final diagnosis and CE PET/CT distinguished N0, N1a, and N1b (weighted kappa [ $\mathrm{k}], 0.704$ ), whereas the final diagnosis and neck CT showed moderate agreement (weighted $\kappa, 0.5$ ). The accuracies of differentiating N0, N1a, and N1b were $81.2 \%$ for CE PET/CT and $68.2 \%$ for neck CT $(P=0.078)$ (Table 2$)$.

\section{Analysis of each cervical level: contralateral or ipsilateral}

We analyzed the difference in findings between CE PET/CT and neck CT between each level of ipsilateral or contralateral cervical levels. Table 2 describes the results. Cervical levelVI and ipsilateral level IV showed significantly different findings between CE PET/CT and neck CT ( $P<0.05$ in all comparisons). In the other cervical levels, there was not significant difference between CE PET/CT and neck CT. In both levels of VI and ipsilateral IV, CE PET/CT was significantly more sensitive $(86.7 \%$ vs. $40.0 \%$ in ipsilateral level IV; $52.9 \%$ vs. $29.4 \%$ in level VI) than neck CT. Also, CE PET/CT showed higher NPV (97.1\% vs. $88.3 \%$ in ipsilateral level IV; $75.0 \%$ vs. $66.7 \%$ in level VI) for detecting metastatic LNs than neck CT (Table 2).

\section{Analysis by number of cervical levels involved}

CE PET/CT and the final diagnosis showed good agreement after calculating the number of cervical levels with a LN metastasis (weighted $\kappa, 0.692$ ). Neck $\mathrm{CT}$ and the final diagnosis showed moderate agreement (weighted $\kappa, 0.506$ ). CE PET/CT and neck $\mathrm{CT}$ showed good agreement for the number of cervical levels involved (weighted $\kappa, 0.685$ ). However, neck $\mathrm{CT}$ showed fewer cervical levels with a metastasis that that of PET/CT $(P=0.009)$.

\section{Analysis of clinical factors affecting the accuracy of CE PET/ CT and neck CT}

For CE PET/CT, when tumors were in both lobes, CE PET/CT less frequently (both $63.0 \%$ vs. $84.5 \%$ ) staged LNs (NO, N1a, and N1b) correctly than when the tumor was in one lobe, but it failed to show statistical significance $(P=0.053)$. For neck $\mathrm{CT}$, when tumors were in both lobes, neck $\mathrm{CT}$ less frequently (both 
Table 2. Diagnostic performance of CE PET/CT and neck CT in cervical N staging preoperative evaluation of thyroid cancer (differentiation between N0 and N1)

\begin{tabular}{|c|c|c|c|c|c|c|c|c|c|}
\hline Variable & TP & $\mathrm{FP}$ & $\mathrm{FN}$ & TN & Sensitivity $(\%, \mathrm{Cl})$ & Specificity $(\%, \mathrm{Cl})$ & Accuracy (\%) & PPV $(\%, \mathrm{Cl})$ & $\mathrm{NPV}(\%, \mathrm{Cl})$ \\
\hline \multicolumn{10}{|c|}{ By patients (N0, N1) } \\
\hline Neck CT & 17 & 6 & 21 & 41 & $44.7(28.6-61.7)$ & $87.2(74.3-95.2)$ & 68.2 & $73.9(51.6-89.8)$ & $66.1(53.0-77.7)$ \\
\hline CE PET/CT & 25 & 3 & 13 & 44 & $65.8(48.7-80.4)$ & $93.6(82.5-98.7)$ & 81.2 & $89.3(71.8-97.7)$ & $77.2(64.2-87.3)$ \\
\hline$P$-value & & & & & $0.008^{\mathrm{a})}$ & 0.375 & 0.078 & 0.055 & $0.001^{\mathrm{a})}$ \\
\hline \multicolumn{10}{|c|}{ By ipsilateral levels } \\
\hline \multicolumn{10}{|l|}{ I } \\
\hline Neck CT & 1 & 0 & 0 & 84 & $100(2.5-100)$ & $100(95.7-100)$ & 100 & $100(2.5-100)$ & $100(95.7-100)$ \\
\hline CE PET/CT & 0 & 0 & 1 & 84 & $0(0-97.5)$ & $100(95.7-100)$ & 98.8 & NA & 98.8 (93.6-99.9) \\
\hline$P$-value & & & & & NA & NA & 0.998 & NA & NA \\
\hline \multicolumn{10}{|l|}{$\|$} \\
\hline Neck CT & 6 & 3 & 6 & 70 & $50(21.1-78.9)$ & 95.9 (88.5-99.1) & 89.4 & 66.7 (29.9-92.5) & $92.1(83.6-97.2)$ \\
\hline CE PET/CT & 9 & 1 & 3 & 72 & $75(42.8-94.5)$ & $98.6(92.6-100)$ & 95.29 & 90 (55.5-99.8) & 96 (88.9-99.2) \\
\hline$P$-value & & & & & 0.25 & 0.5 & 0.249 & 0.072 & 0.070 \\
\hline \multicolumn{10}{|l|}{ III } \\
\hline Neck CT & 11 & 0 & 8 & 66 & $57.9(33.5-79.8)$ & $100(94.5-100)$ & 90.7 & $100(71.5-100)$ & $89.3(79.8-95.2)$ \\
\hline CE PET/CT & 13 & 1 & 6 & 65 & $68.4(43.5-87.4)$ & $98.5(91.8-100)$ & 91.8 & $92.9(66.1-99.8)$ & $91.6(82.5-96.8)$ \\
\hline$P$-value & & & & & 0.688 & 1.0 & 1.0 & 0.301 & 0.437 \\
\hline \multicolumn{10}{|l|}{ IV } \\
\hline Neck CT & 6 & 2 & 9 & 68 & $40.0(16.3-67.7)$ & $97.1(90.1-99.7)$ & 87.1 & $75.0(34.9-96.8)$ & $88.3(79.0-94.5)$ \\
\hline CE PET/CT & 13 & 4 & 2 & 66 & $86.7(59.5-98.3)$ & $94.4(86.0-98.4)$ & 93.0 & $76.5(50.1-93.2)$ & $97.1(89.8-99.6)$ \\
\hline$P$-value & & & & & $0.016^{a)}$ & 0.620 & 0.367 & 0.911 & $0.006^{a)}$ \\
\hline \multicolumn{10}{|l|}{ V } \\
\hline Neck CT & 0 & 1 & 2 & 82 & $0(0-84.2)$ & $98.8(93.5-100)$ & 96.5 & $0(0-97.5)$ & 97.6 (91.7-99.7) \\
\hline CE PET/CT & 1 & 0 & 1 & 83 & $50(1.3-98.7)$ & $100(95.7-100)$ & 98.8 & $100(2.5-100)$ & $98.8(93.5-100)$ \\
\hline$P$-value & & & & & 1.0 & 1.0 & 0.614 & 0.157 & 0.309 \\
\hline \multicolumn{10}{|l|}{ VI (central) } \\
\hline Neck CT & 10 & 3 & 24 & 48 & $29.4(15.1-47.5)$ & $94.1(83.8-98.8)$ & 68.2 & $76.9(46.2-95.0)$ & $66.7(54.6-77.3)$ \\
\hline CE PET/CT & 18 & 3 & 16 & 48 & $52.9(35.1-70.2)$ & $94.1(83.8-98.8)$ & 77.7 & $85.7(63.7-97.0)$ & $75.0(62.6-85.0)$ \\
\hline$P$-value & & & & & $0.008^{a)}$ & 1.0 & 0.227 & 0.312 & $0.004^{a)}$ \\
\hline \multicolumn{10}{|l|}{ VII } \\
\hline Neck CT & 3 & 2 & 1 & 79 & 75 (19.4-99.4) & $97.5(91.4-99.7)$ & 96.5 & $60(14.7-94.7)$ & 98.8 (93.2-99.9) \\
\hline CE PET/CT & 3 & 2 & 1 & 79 & $75(19.4-99.4)$ & $97.5(91.4-99.7)$ & 96.5 & $60(14.7-94.7)$ & $98.8(93.2-99.9)$ \\
\hline$P$-value & & & & & NA & 1.0 & 0.678 & 1.0 & 1.0 \\
\hline \multicolumn{10}{|c|}{ By contralateral levels ${ }^{\text {b) }}$} \\
\hline \multicolumn{10}{|l|}{ I } \\
\hline Neck CT & 0 & 0 & 0 & 58 & NA & $100(93.8-100)$ & 100 & NA & $100(93.8-100)$ \\
\hline CE PET/CT & 0 & 1 & 0 & 57 & NA & $98.3(90.8-100)$ & 98.3 & $0(0-97.5)$ & $100(93.7-100)$ \\
\hline$P$-value & & & & & NA & 1.0 & 0.989 & NA & NA \\
\hline \multicolumn{10}{|l|}{$\|$} \\
\hline Neck CT & 0 & 1 & 1 & 56 & $0(0-97.5)$ & $98.3(90.6-100)$ & 95.6 & $0(0-97.5)$ & $98.3(90.6-100)$ \\
\hline CE PET/CT & 0 & 0 & 1 & 57 & $0(0-97.5)$ & $100(93.7-100)$ & 98.3 & NA & $98.3(90.8-100)$ \\
\hline$P$-value & & & & & NA & 1.0 & 0.760 & NA & NA \\
\hline \multicolumn{10}{|l|}{ III } \\
\hline Neck CT & 0 & 0 & 0 & 58 & NA & $100(93.8-100)$ & 100 & NA & $100(93.8-100)$ \\
\hline CE PET/CT & 0 & 0 & 0 & 58 & NA & $100(93.8-100)$ & 100 & NA & $100(93.8-100)$ \\
\hline$P$-value & & & & & NA & NA & NA & NA & NA \\
\hline \multicolumn{10}{|l|}{ IV } \\
\hline Neck CT & 1 & 1 & 0 & 56 & $100(2.5-100)$ & $98.25(90.6-100)$ & 98.3 & $50(1.3-98.7)$ & $100(93.6-100)$ \\
\hline CE PET/CT & 1 & 0 & 0 & 57 & $100(2.5-100)$ & $100(93.7-100)$ & 100 & $100(2.5-100)$ & $100(93.7-100)$ \\
\hline$P$-value & & & & & NA & 1.0 & 0.989 & 0.18 & NA \\
\hline
\end{tabular}


Table 2. Continued

\begin{tabular}{|c|c|c|c|c|c|c|c|c|c|}
\hline Variable & TP & $\mathrm{FP}$ & FN & $\mathrm{TN}$ & Sensitivity $(\%, \mathrm{Cl})$ & Specificity $(\%, \mathrm{Cl})$ & Accuracy (\%) & $\mathrm{PPV}(\%, \mathrm{Cl})$ & $\mathrm{NPV}(\%, \mathrm{Cl})$ \\
\hline \multicolumn{10}{|l|}{ V } \\
\hline Neck CT & 0 & 0 & 0 & 58 & NA & $100(93.8-100)$ & 100 & NA & $100(93.84-100)$ \\
\hline CE PET/CT & 0 & 0 & 0 & 58 & NA & $100(93.8-100)$ & 100 & NA & $100(93.8-100)$ \\
\hline$P$-value & & & & & NA & NA & NA & NA & NA \\
\hline \multicolumn{10}{|l|}{ By neck side } \\
\hline \multicolumn{10}{|c|}{ Ipsilateral side ${ }^{c)}$} \\
\hline Neck CT & 13 & 2 & 6 & 64 & $68.4(43.5-87.4)$ & $97.0(89.5-99.6)$ & 90.6 & 86.7 (59.5-98.3) & $91.4(82.3-96.8)$ \\
\hline CE PET/CT & 16 & 2 & 3 & 64 & $84.2(60.4-96.6)$ & $97.0(89.5-99.6)$ & 94.1 & $88.9(65.3-98.6)$ & $95.5(87.5-99.1)$ \\
\hline$P$-value & & & & & 0.25 & 1.0 & 0.569 & 0.838 & 0.078 \\
\hline \multicolumn{10}{|c|}{ Contralateral side ${ }^{d)}$} \\
\hline Neck CT & 1 & 1 & 1 & 55 & $50.0(1.4-98.7)$ & $98.2(90.5-99.9)$ & 96.6 & $50.0(1.4-98.7)$ & $98.21(90.5-99.9)$ \\
\hline CE PET/CT & 1 & 1 & 1 & 55 & $50.0(1.4-98.7)$ & $98.2(90.5-99.9)$ & 96.6 & $50.0(1.4-98.7)$ & 98.21 (90.5-99.9) \\
\hline$P$-value & & & & & NA & 1.0 & 0.608 & 1.0 & 1.0 \\
\hline
\end{tabular}

CE PET/CT, ${ }^{18} \mathrm{~F}$-fluorodeoxyglucose positron emission tomography/computed tomography with contrast-enhancement; TP, true-positive; FP, false-positive; FN, false-negative; TN, true-negative; $\mathrm{Cl}$, confidence interval; PPV, positive predictive value; NPV, negative predictive value; NA, not available. a) $P<0.05$. b-d) For analysis of "contralateral level or side," we analyzed 58 patients with unilateral tumor. For patients with bilateral tumors ( $\mathrm{n}=27)$, metastastic lymph nodes in either side of the neck were analyzed as ipsilateral side.

Table 3. Comparison of clinical factors and final results of lymph node staging by PET or CT

\begin{tabular}{|c|c|c|c|c|c|c|}
\hline \multirow{2}{*}{ Variable } & \multicolumn{3}{|c|}{ CE PET/CT staging } & \multicolumn{3}{|c|}{ Neck CT } \\
\hline & Correct & Incorrect & $P$-value & Correct & Incorrect & $P$-value \\
\hline Age (yr) & $53.2 \pm 10.2$ & $50.2 \pm 10.2$ & 0.331 & $53.5 \pm 12.6$ & $50.26 \pm 9.85$ & 0.236 \\
\hline Sex (male:female) & $14: 52$ & $2: 17$ & 0.506 & $13: 45$ & $3: 24$ & 0.251 \\
\hline PET/CT machine model (A:B:C:D:E) & $33: 8: 13: 7: 5$ & $11: 1: 4: 3: 0$ & 0.579 & $32: 8: 9: 5: 4$ & $12: 1: 8: 5: 1$ & 0.209 \\
\hline PET arm position (up:down) & $42: 24$ & $12: 7$ & 0.816 & 36:22 & $18: 9$ & 0.867 \\
\hline Tumor size $(\mathrm{cm})$ & $0.8(0.2-5.0)$ & $0.9(0.4-2.8)$ & 0.321 & $0.8(0.2-5.0)$ & $1.1(0.3-3.8)$ & 0.062 \\
\hline $\begin{array}{l}\text { Extra-thyroidal extension } \\
\text { (none:micro:macro) }\end{array}$ & $26: 33: 7$ & $6: 13: 0$ & 0.861 & $23: 29: 6$ & $9: 17: 1$ & 0.982 \\
\hline $\begin{array}{l}\text { Tumor location } \\
\text { (unilateral:bilateral) }\end{array}$ & $49: 17$ & 9:10 & 0.053 & $42: 16$ & $16: 11$ & 0.336 \\
\hline No. of primary tumors & $1(1-7)$ & $2(1-5)$ & $0.016^{a)}$ & $1(1-7)$ & $2(1-5)$ & $0.136^{a)}$ \\
\hline $\begin{array}{l}\text { No. of patients with multiple tumors } \\
(1: 2: 3: 4: 5: 6 \text { tumors })\end{array}$ & $44: 16: 3: 2: 0: 1$ & $6: 11: 1: 1: 0: 0$ & $0.099^{b)}$ & $38: 14: 3: 2: 0: 1$ & 12:13:1:1:0:0 & $0.441^{\mathrm{b}}$ \\
\hline Primary tumor SUVmax & $3.9(1.5-49.5)$ & $6.0(2.2-50.7)$ & 0.087 & $3.9(1.5-48.5)$ & $5.5(1.7-50.68)$ & 0.199 \\
\hline Interval from scan to operation (day) & $18.5(1-78)$ & $22(0-62)$ & 0.804 & $18.5(1-78)$ & $22(0-64)$ & 0.610 \\
\hline Institution (A:B:C:D) & $41: 11: 9: 5$ & $12: 3: 4: 0$ & 0.58 & $10: 6: 8: 4$ & $13: 8: 5: 1$ & 0.110 \\
\hline
\end{tabular}

Values are presented as mean \pm standard deviation (age) or median with range (tumor size, number of primary tumors, SUVmax, and interval). For analysis, we used groups of "correct"/"incorrect" cases of each modalities based on the final results which were determined by surgical biopsy and clinical follow-up till postoperative 6 months. For example, when CE PET/CT successfully staged the LN (NO, N1a, and N1b) in some patients, they were categorized as CE PET/CT correct cases.

PET, positron emission tomography; CT, computed tomography; CE PET/CT, ${ }^{18}$ F-fluorodeoxyglucose positron emission tomography/computed tomography with contrast-enhancement; SUVmax, maximum standardized uptake value.

a)Mann-Whitney test. ${ }^{\text {b) }}$ Chi-square for trend.

$59.3 \%$ vs. $72.4 \%$ ) staged LNs (N0, N1a, and N1b) correctly than when the tumor was in one lobe, but it also failed to show statistical significance $(P=0.336)$.

The primary tumor SUVmax values were higher when tumors were on bilateral lobes (median, 10.8) than when a tumor was on one lobe (median, $3.75 ; P=0.000$ ). However, no difference in the primary tumor SUVmax was found between patients whose $\mathrm{CE}$ PET/CT scan was correct and whose CE PET/ $\mathrm{CT}$ scan was incorrect in distinguishing N0, N1a, and N1b (same result as when differentiating N0 or N1). Partial correlation failed to show significant effect of tumor side (ipsilateral vs. bilateral) and SUVmax on the accuracy of NE PET/CT. The logistic regression also failed to show a significant effect of primary tumor SUVmax on the accuracy of CE PET/CT or neck CT.

Other clinical factors, including sex, scanner type, arm position, extrathyroidal extension, pathologic tumor type, age, tumor size, intervals between CE PET/CT and operation, or neck CT to the operation, or CE PET/CT to neck CT did not affect 
the accuracy of CE PET/CT or neck CT (Table 3). The median value of number of tumors of CE PET/CT correct cases were lower than that of incorrect case $(P=0.016)$. However, chisquare for trend failed to show tendency of smaller or larger number of tumor were related to be PET/CT N staging accuracy. In addition, no significant differences were found relating to the accuracies for LN staging by CE PET/CT and neck CT at any of the institutions.

\section{DISCUSSION}

Our results suggest that CE PET/CT has higher sensitivity and NPV than neck CT for preoperative LN staging in patients with thyroid cancer. By level-by-level analysis, only level VI and ipsilateral level IV showed statistically significant differences in sensitivity and NPV between CE PET/CT and neck CT in N staging. Jeong et al. [15] reported that NE PET/CT has sensitivity of $30.4 \%$, specificity of $96.2 \%$, and diagnostic accuracy of $86.9 \%$ at all LN levels. In their study, the corresponding values for neck CT were $34.8 \%, 96.2 \%$, and $87.2 \%$ (CE CT). Kim et al. [8] reported that preoperative LN staging of patients with papillary thyroid cancer by neck CT has sensitivity of $62 \%$ and specificity of $93 \%$. Pak et al. reviewed related studies and reported that sensitivity and specificity of PET/CT for central LNs were 5.0\% to $22.7 \%$ and $93.6 \%$ to $98.8 \%$, respectively; and those of CE CT were $15.1 \%$ to $25.0 \%$ and $93.8 \%$ to $98.8 \%$, respectively $[10,15,17-20]$. Sensitivity and specificity of PET for lateral LNs were $30.6 \%$ to $50 \%$ and $90.4 \%$ to $97 \%$, respectively; and those of CE CT were $33.3 \%$ to $42.3 \%$ and $53.6 \%$ to $96.6 \%$, respectively $[10,15,17-20]$. In their report, PET/CT and CE CT specificities were $>90 \%$ for central and lateral neck LNs $[10,15,17-20]$. Based on these data, the frequency of distant metastasis at thyroid cancer staging is only $4 \%$ to $7 \%$, and surgery is the only therapeutic option for thyroid cancer. Pak et al. [10] reported that routine use of PET/CT for a preoperative evaluation is inappropriate, except in patients with aggressive pathologies, who have a higher risk for distant metastasis [2123]. However, these data are all from NE PET/CT. Our CE PET/ CT sensitivity was much higher than that of previous studies $[13,15,17-20,24]$, which may be due to the additional information obtained from CE CT in our study. No study has compared preoperative thyroid cancer staging by CE PET/CT and NE PET/CT. One study on pancreatic cancer by Yoneyama et al. [24] reported that the diagnostic accuracies of distant metastasis, scalene node metastasis, and peritoneal dissemination on CE PET/ CT were significantly higher than those for NE PET/CT $(P<0.05)$ [24]. A comparison of LN staging between CE PET/ $\mathrm{CT}$ and NE PET/CT is beyond the scope of this study; thus, additional studies are needed.

In our study, bilateral tumors have higher primary tumor SUVmax values. We thought that higher FDG uptake might make it difficult to detect FDG uptake into adjacent LNs, which would affect diagnostic accuracy. However, there was no significant difference in accuracy of CE PET/CT between cases with bilateral tumors and cases with ipsilateral tumors $(P=0.053)$. In addition, there was no significant difference was observed in the primary tumor SUVmax between the PET/CT correct group and incorrect groups, and the logistic regression failed to show a significant result. Byun et al. [11] reported that tumor SUVmax values $>2.8$ are associated with central LN metastasis in a study on microcarcinoma of the thyroid. However, their study focused on predicting LN metastasis and not on detection. No study has analyzed the relationship between tumor SUVmax or tumor multiplicity and detectability of LN metastasis by PET/CT.

Several limitations of our study should be mentioned. First, although we followed up for 6 months, false-negative cases could have occurred, which were beyond the operation field range. Second, we retrospectively analyzed the findings from scan reports at each of the institutions without additional reading; thus, bias may have occurred.Third, the number of the cases with LN metastasis was small in some cervical levels. In addition, we only analyzed CE PET/CT without comparing CE PET/ $\mathrm{CT}$ and NE PET/CT.

In conclusion, ${ }^{18} \mathrm{~F}$-FDG PET/CT with CE CT was more sensitive and reliable than neck $\mathrm{CT}$ for preoperative $\mathrm{LN}$ staging of patients with thyroid cancer. Considering that torso PET/CT also provides information about regions beyond the cervical area, applying PET/CT with CE CT is advantageous for preoperatively evaluating thyroid cancer.

\section{CONFLICT OF INTEREST}

No potential conflict of interest relevant to this article was reported.

\section{ACKNOWLEDGMENTS}

This is a clinical study of the Korean Society of Nuclear Medicine Imaging Network for Clinical Trials (KSNM CTN) supported by the Korean Society of Nuclear Medicine (KSNM-CTN2014-01-1).

\section{REFERENCES}

1. Siegel R, Naishadham D, Jemal A. Cancer statistics, 2012. CA Cancer J Clin. 2012 Jan-Feb;62(1):10-29.

2. Enewold L, Zhu K, Ron E, Marrogi AJ, Stojadinovic A, Peoples GE, et al. Rising thyroid cancer incidence in the United States by demographic and tumor characteristics, 1980-2005. Cancer Epidemiol Biomarkers Prev. 2009 Mar;18(3):784-91.

3. Dimov RS. The effect of neck dissection on quality of life in patients 
with differentiated thyroid cancer. Gland Surg. 2013 Nov;2(4):21926.

4. Adam MA, Pura J, Goffredo P, Dinan MA, Reed SD, Scheri RP, et al. Presence and number of lymph node metastases are associated with compromised survival for patients younger than age 45 years with papillary thyroid cancer. J Clin Oncol. 2015 Jul;33(21):2370-5.

5. Stack BC Jr, Ferris RL, Goldenberg D, Haymart M, Shaha A, Sheth S, et al. American Thyroid Association consensus review and statement regarding the anatomy, terminology, and rationale for lateral neck dissection in differentiated thyroid cancer.Thyroid. 2012 May;22(5): 501-8.

6. Luster M,WeberT, Verburg FA. Differentiated thyroid cancer-personalized therapies to prevent overtreatment. Nat Rev Endocrinol. 2014 Sep;10(9):563-74.

7. Grant CS. Papillary thyroid cancer: strategies for optimal individualized surgical management. Clin Ther. 2014 Jul;36(7):1117-26.

8. Kim E, Park JS, Son KR, Kim JH, Jeon SJ, Na DG. Preoperative diagnosis of cervical metastatic lymph nodes in papillary thyroid carcinoma: comparison of ultrasound, computed tomography, and combined ultrasound with computed tomography. Thyroid. 2008 Apr;18(4):411-8.

9. Langer JE, Mandel SJ. Sonographic imaging of cervical lymph nodes in patients with thyroid cancer. Neuroimaging Clin N Am. 2008 Aug;18(3):479-89.

10. Pak K, Kim SJ, Kim IJ, Kim BH, Kim SS, Jeon YK. The role of 18Ffluorodeoxyglucose positron emission tomography in differentiated thyroid cancer before surgery. Endocr Relat Cancer. 2013 Jul;20(4): R203-13.

11. Byun BH, Jeong UG, Hong SP, Min JJ, Chong A, Song HC, et al. Prediction of central lymph node metastasis from papillary thyroid microcarcinoma by $18 \mathrm{~F}$-fluorodeoxyglucose PET/CT and ultrasonography. Ann Nucl Med. 2012 Jul;26(6):471-7.

12. Choi JS, Kim J, Kwak JY, Kim MJ, Chang HS, Kim EK. Preoperative staging of papillary thyroid carcinoma: comparison of ultrasound imaging and CT.AJR Am J Roentgenol. 2009 Sep;193(3):871-8.

13. American Joint Committee on Cancer. AJCC cancer staging manual. 6th ed. New York: Springer-Verlag; 2002.

14. Som PM. Detection of metastasis in cervical lymph nodes: CT and MR criteria and differential diagnosis. AJR Am J Roentgenol. 1992 May;158(5):961-9.

15. Jeong HS, Baek CH, Son YI, Choi JY, Kim HJ, Ko YH, et al. Integrat- ed 18F-FDG PET/CT for the initial evaluation of cervical node level of patients with papillary thyroid carcinoma: comparison with ultrasound and contrast-enhanced CT. Clin Endocrinol (Oxf). 2006 Sep;65(3):402-7.

16. Mitchell JC, Grant F, Evenson AR, Parker JA, Hasselgren PO, Parangi S. Preoperative evaluation of thyroid nodules with 18FDG-PET/ CT. Surgery. 2005 Dec;138(6):1166-74.

17. ChoiWH, Chung YA, Han EJ, Sohn HS, Lee SH. Clinical value of integrated [18F]fluoro-2-deoxy-D-glucose positron emission tomography/computed tomography in the preoperative assessment of papillary thyroid carcinoma: comparison with sonography. J Ultrasound Med. 2011 Sep;30(9):1267-73.

18. Choi JW, Yoon YH, Yoon YH, Kim SM, Koo BS. Characteristics of primary papillary thyroid carcinoma with false-negative findings on initial (18)F-FDG PET/CT. Ann Surg Oncol. 2011 May;18(5):130611.

19. Jeong HS, Chung M, Baek CH, Ko YH, Choi JY, Son YI. Can [18F]fluorodeoxyglucose standardized uptake values of PET imaging predict pathologic extrathyroid invasion of thyroid papillary microcarcinomas? Laryngoscope. 2006 Dec;116(12):2133-7.

20. Morita S, Mizoguchi K, Suzuki M, lizuka K. The accuracy of (18)[F]fluoro-2-deoxy-D-glucose-positron emission tomography/computed tomography, ultrasonography, and enhanced computed tomography alone in the preoperative diagnosis of cervical lymph node metastasis in patients with papillary thyroid carcinoma. World J Surg. 2010 Nov;34(11):2564-9.

21. Schlumberger M,Tubiana M, De Vathaire F, Hill C, Gardet P,Travagli JP, et al. Long-term results of treatment of 283 patients with lung and bone metastases from differentiated thyroid carcinoma. J Clin Endocrinol Metab. 1986 Oct;63(4):960-7.

22. Shaha AR, Shah JP, Loree TR. Differentiated thyroid cancer presenting initially with distant metastasis. Am J Surg. 1997 Nov;174(5): 474-6.

23. Pryma DA, Schoder H, Gonen M, Robbins RJ, Larson SM, Yeung HW. Diagnostic accuracy and prognostic value of 18F-FDG PET in Hurthle cell thyroid cancer patients. J Nucl Med. 2006 Aug;47(8): 1260-6.

24. Yoneyama T, Tateishi U, Endo I, Inoue T. Staging accuracy of pancreatic cancer: comparison between non-contrast-enhanced and contrast-enhanced PET/CT. Eur J Radiol. 2014 Oct;83(10):1734-9. 